\title{
A New Index AGR-PLR Score (APS) for Patients with Esophageal Squamous Cell Carcinoma Undergoing Radical Esophagectomy
}

\section{Yimin Yao \\ Chang Cai \\ Fei Sun \\ Wei Gong}

Department of Radiation Oncology, The First Affiliated Hospital of Soochow University, Suzhou, 215000, People's Republic of China
Correspondence: Wei Gong

Department of Radiation Oncology, The

First Affiliated Hospital of Soochow

University, Suzhou, 215000, People's

Republic of China

$\mathrm{Tel}+86-512-67780008$

Email20184|32209@stu.suda.edu.cn
Purpose: Biomarkers of the systemic inflammatory response and nutritional-related indicators have been used to assess the host anti-tumor immune response and predict prognosis in esophageal squamous cell carcinoma (ESCC). However, a new indicator system combining platelet-to-lymphocyte ratio (PLR) and albumin-globulin ratio (AGR), AGR-PLR score (APS), has not yet been evaluated for the prognosis prediction among ESCC patients.

Methods: A retrospective analysis was performed, including 633 patients with ESCC, comprising 450 in the training cohort and 183 in the validation cohort.

Results: In this study, we found that the overall survival time among patients with an APS of 2 was significantly shorter than that among patients with an APS of 1, and the survival time of patients with an APS of 1 was significantly shorter than that of patients with an APS of 0. Multivariate analysis showed that the APS was an independent prognostic factor for patients with ESCC. The APS demonstrated better prognostic accuracy and effectiveness for ESCC patients than either PLR or AGR alone. In addition, a new prediction nomogram was established according to tumor grade, APS, and tumor, node, metastasis (TNM) stage. Compared with the traditional 8th version of TNM staging system, this nomogram demonstrated improved sensitivity and specificity for the prediction of 3- and 5-year survival.

Conclusion: APS is a novel independent prognostic indicator for the radical resection of ESCC and a potential biomarker for monitoring the therapeutic response.

Keywords: AGR, ESCC, nomogram, prognosis, PLR

\section{Introduction}

Esophageal cancer (EC) is one of the most common malignant tumors that affect the digestive system, characterized by high malignancy, strong invasiveness, and poor prognosis, and it is considered among the leading causes of cancer-related death worldwide. ${ }^{1}$ In China, esophageal squamous cell carcinoma (ESCC) is the most commonly occurring form of EC, accounting for approximately $90 \%$ of all EC cases, whereas esophageal adenocarcinoma is rarer. ${ }^{2,3}$ Currently, clinical treatments for EC include surgical resection and combination treatments of chemotherapy, radiotherapy, and immunotherapy. ${ }^{4}$ However, despite recent advances in the diagnosis and comprehensive treatment of ESCC, the prognosis of ESCC patients remains poor, with a 5-year survival rate of only $10 \%-30 \%{ }^{5}$ At present, prognosis and treatments for ESCC patients are primarily guided by the tumor, node, metastasis (TNM) staging system. However, differentiated survival rates across ESCC patients with the same TNM stage who receive the same treatment have been 
observed in clinic. Therefore, a new clinical prognostic indicator for ESCC that can be used in tandem with the TNM staging system is urgently necessary to provide effective prognostic predictions and treatment guidance to further limit tumor progression and promote survival among ESCC patients.

Growing evidence indicates that interactions between inflammation and immune responses play key roles in the tumorigenesis, proliferation, progression, and metastasis of malignant tumors. ${ }^{6}$ Systemic inflammatory response biomarkers, such as the neutrophil-to-lymphocyte ratio (NLR), the platelet-to-lymphocyte ratio (PLR), and the monocyte-to-lymphocyte ratio (MLR), have been used to assess the host anti-tumor immune responses and predict prognosis in multiple malignant tumors, including ESCC. ${ }^{7,8}$ Recently, several studies have reported the use of the serum albumin-globulin ratio (AGR), a nutrition-related indicator, to predict prognosis in patients with multiple tumors. ${ }^{9,10}$ However, in ESCC, the AGR-PLR score (APS), a new indicator that combines systemic inflammatory factors with nutritional-related indicators, has not yet been evaluated for prognosis prediction among ESCC patients. Therefore, in this study, we aimed to explore the ability of the APS to predict postoperative prognosis among ESCC patients established a nomogram based on APS and TNM stages to provide better ESCC prognostic predictions.

\section{Materials and Methods}

\section{Participants}

A retrospective analysis was performed using the clinical pathology data from 450 ESCC who were patients admitted and received surgeries at the First Affiliated Hospital of Soochow University from 2011 to 2015. The analysis results were then confirmed in $183 \mathrm{ESCC}$ who were patients admitted and received surgery from 2016 to 2019. Inclusion criteria included: 1) ESCC confirmed by postoperative pathology; 2) no preoperative chemotherapy, radiotherapy, immunotherapy, or other complementary treatment; 3) no distant metastasis at diagnosis; 4) no severe liver or kidney dysfunction, acute or chronic infection, malnutrition markers detected in the blood or immune system, or other diseases affecting indicators of blood cells, albumin, or coagulation before surgery; 5) received radical resection of EC; and 6) complete data, including clinical pathology, laboratory examination and follow-up. Follow-up studies to determine the postoperative survival status of patients were performed through outpatient or telephone review, conducted once every 3 months during the first year and once every 6 months starting in the second year after surgery and continuing until December 2019. Overall survival (OS) was defined as the duration between the surgery date and date of death or the last valid follow-up. This study was performed in accordance with the Helsinki Declaration. This study was approved by the Ethics Committee of the First Affiliated Hospital of Soochow University. All patients in this study signed informed consent forms.

\section{Peripheral Blood Collection and Related Indicators Calculation}

Morning peripheral venous blood samples were collected from ESCC patients 1 week before surgery. Neutrophil, lymphocyte and platelet counts were obtained from routine tests using an automatic blood cell analyzer (Sysmex XN9000, Japan). Serum albumin and globulin levels were detected using an automated biochemical analyzer (Beckman, USA). NLR, MLR, PLR and AGR were calculated according to the following equations: NLR = peripheral blood neutrophil count/peripheral lymphocyte count; PLR $=$ peripheral platelet count/peripheral blood lymphocyte count; MLR = peripheral blood monocyte count/peripheral blood lymphocyte count; and AGR = the absolute value of peripheral albumin/the absolute value of peripheral globulin. The optimal cut-off values for NLR, PLR, MLR, and AGR were determined according to receiver operating characteristic curve (ROC): NLR (NLR $\leq 1.36$, NLR $>$ 1.36), PLR (PLR $\leq 132$, PLR $>132)$, MLR $(M L R \leq 0.27$, MLR > 0.27), and AGR (AGR $\leq 1.75$, AGR > 1.75).

\section{Statistical Analysis}

The correlation between APS and the clinicopathological parameters of ESCC patients was evaluated using the Chisquare test. Kaplan-Meier analysis were used for survival analysis. Multivariate survival analyses were conducted using the Cox Proportional Risk Model. The predictive efficacies of independent prognostic factors were evaluated and compared using the area under the time-dependent ROC-curve (AUC). The nomogram was established in accordance with the Akaike Information Guidelines. Bootstrap repeat sampling (1000 bootstrap re-samplings) was used for the verification and calibration of the nomogram. All statistical analyses were conducted using SPSS 22.0 (Chicago, IL, USA), Graph Pad Prism 6 (La Jolla, CA, USA) and R 3.0.3 Software (Vienna, Austria). $P<0.05$ was considered significant. 


\section{Results}

Correlations Between Systemic Inflammatory Index, Nutritional Index, and ESCC Patient Prognosis

A total of 633 patients with ESCC were included in this study, including 450 in the original cohort and 183 in the validation cohort. The baseline clinical and demographic data for patients in both the original and validation cohorts are displayed in Table 1. In the original cohort, the optimal cut-off values of PLR, NLR, MLR, and AGR for ESCC patient

Table I Clinicopathological Characteristics of ESCC Patients

\begin{tabular}{|c|c|c|c|c|}
\hline \multirow[t]{2}{*}{ Characteristic } & \multicolumn{2}{|c|}{$\begin{array}{l}\text { Original Queue } \\
\qquad(n=450)\end{array}$} & \multicolumn{2}{|c|}{$\begin{array}{l}\text { Validation Queue } \\
\qquad(n=183)\end{array}$} \\
\hline & $\begin{array}{c}\text { No. of } \\
\text { Patients }\end{array}$ & $\%$ & $\begin{array}{c}\text { No. of } \\
\text { Patients }\end{array}$ & $\%$ \\
\hline \multicolumn{5}{|l|}{ Sex } \\
\hline Male & 343 & 76.4 & 142 & 77.6 \\
\hline Female & 107 & 23.6 & 41 & 22.4 \\
\hline \multicolumn{5}{|l|}{ Age } \\
\hline$\leq 60$ & 210 & 46.7 & 72 & 39.3 \\
\hline$>60$ & 240 & 53.3 & 111 & 60.4 \\
\hline \multicolumn{5}{|l|}{ Tumor location } \\
\hline Upper & 30 & 6.7 & 8 & 4.3 \\
\hline Middle & 270 & 60.0 & 111 & 60.7 \\
\hline Lower & 150 & 33.3 & 64 & 35.0 \\
\hline \multicolumn{5}{|l|}{ Histological grade } \\
\hline Well differentiated & 26 & 5.8 & 15 & 8.2 \\
\hline Moderately & 198 & 44.0 & 95 & 51.9 \\
\hline differentiated & & & & \\
\hline $\begin{array}{l}\text { Poorly or not } \\
\text { differentiated }\end{array}$ & 226 & 50.2 & 73 & 39.9 \\
\hline \multicolumn{5}{|l|}{ T stage } \\
\hline TI & 106 & 23.5 & 49 & 26.8 \\
\hline $\mathrm{T} 2$ & 120 & 26.7 & 45 & 24.6 \\
\hline T3 & 213 & 47.3 & 89 & 48.6 \\
\hline $\mathrm{T} 4 \mathrm{a}$ & 11 & 2.4 & 0 & 0.00 \\
\hline \multicolumn{5}{|l|}{$\mathrm{N}$ stage } \\
\hline No & 241 & 53.6 & 66 & 36.1 \\
\hline $\mathrm{NI}$ & 134 & 29.8 & 72 & 39.3 \\
\hline N2 & 62 & 13.8 & 37 & 20.2 \\
\hline N3 & 13 & 2.9 & 8 & 4.4 \\
\hline \multicolumn{5}{|l|}{ TNM stage (AJCC, 8th) } \\
\hline 1 & 78 & 17.3 & 38 & 20.8 \\
\hline II & 215 & 47.8 & 50 & 27.3 \\
\hline III & 157 & 34.9 & 95 & 52.0 \\
\hline
\end{tabular}

Abbreviations: ESCC, esophageal squamous cell carcinoma; TNM, tumor, node, metastasis; AJCC, American Joint Committee on Cancer. survival prediction were determined based on the ROC curve, and the results were $132,1.36,0.27$, and 1.75 , respectively. All patients were divided into high-level and low-level groups according to the optimal cut-off values. Using Kaplan-Meier survival analysis, we found that patients with high levels of PLR, NLR, and MLR and low levels of AGR were associated with shorter survival times (Figure 1A-D, all $P<0.05)$. Univariate analysis was subsequently conducted to investigate which clinicopathological variables were associated with postoperative survival among ESCC patients in the original cohort. Tumor grade, TNM stage, PLR, NLR, MLR and AGR were identified as potential to be the risk factors affecting the prognosis of ESCC patients (Table 2). Significant clinicopathological variables identified by the univariate analysis were then incorporated into the multivariate Cox proportional hazard model to determine independent prognostic factors. Tumor grade, TNM stage, PLR, and AGR were identified as independent risk factors affecting the OS of ESCC patients based on the multivariate analysis, whereas NLR and MLR were not identified as independent prognostic factors associated with OS. According to these results, we established the APS system, which combines the PLR and AGR. The APS system included 3 categories ( 0,1 and 2$)$, as follows: APS of 0, PLR $\leq 132$ and AGR $>1.75$; APS of 1 , PLR > 132 or AGR $\leq 1.75$; APS of $2, \mathrm{PLR}>132$ and $\mathrm{AGR} \leq$ 1.75. The correlations between the APS and clinicopathological variables among patients with ESCC are shown in Table 3. APS was associated with PLR, NLR, MLR and AGR, but not with age, sex, grade, location, $\mathrm{T}$ stage, $\mathrm{N}$ stage, or TNM stage (Table 3). A Kaplan-Meier survival analysis was performed for the established APS system. We found that OS among patients with APS of 2 was significantly shorter than that among patients with APS of 1, and the OS among patients with APS of 1 was significantly shorter than that among patients with APS of 0 (Figure 2A). Multivariate analysis was performed, which showed that APS was an independent prognostic factor for patients with ESCC (Table 2). Further analysis of the AUC predicted the 3- and 5-year survival among ESCC patients in the original cohort. We also found APS was associated with a larger AUC for the estimation of prognosis among ESCC patients compared with those for either PLR or AGR alone (Figure 2B and C). Similar results were obtained in the validation cohort (Figure 2D-F).

\section{Nomogram Establishment and Prognosis Performance Assessment}

To further investigate the prognostic value of the APS system, we aimed to extract the optimal predictive model 

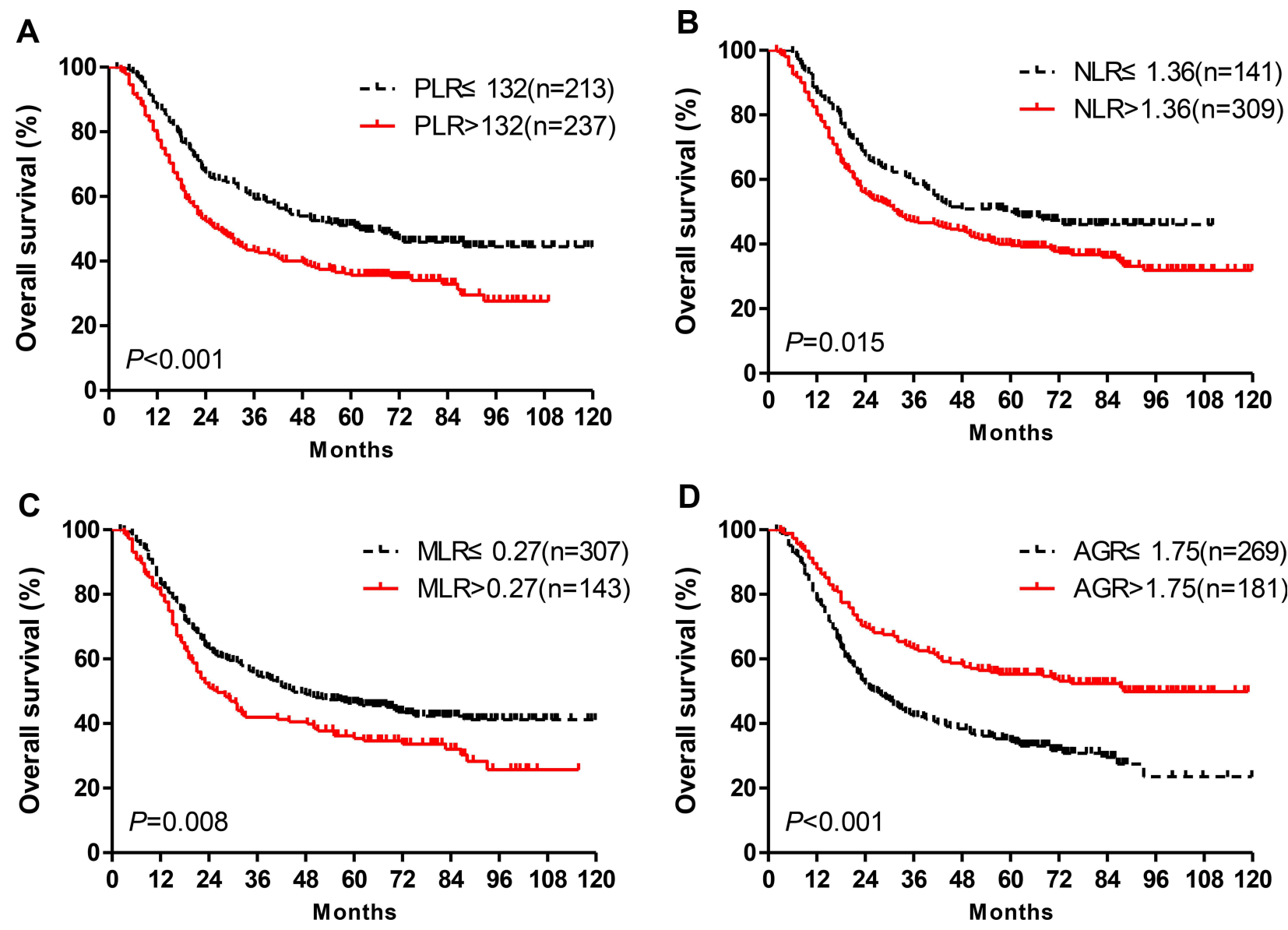

Figure I The prognostic significance is based on the PLR (A), NLR (B), MLR (C) and AGR (D) in ESCC patients from the original cohort.

from the original cohort based on the Cox proportional hazard regression modeling method. Subsequently, a nomogram was established, including tumor grade, APS, and TNM stage for the prediction of 3- and 5-year survival among ESCC patients (Figure 3). The C-index of the nomogram was 0.80 , which was significantly higher than that of TNM $(0.73, P<0.001)$. The calibrated 3- and 5 -year survival rates indicated that the nomogram predictions were highly coordinated with real-world observations (Figure 4A and B). We also investigated differences in the prognostic prediction capabilities between the established nomogram and the traditional TNM staging system using AUC analysis. We found that the AUC of the established nomogram was larger than that for the TNM staging system (Figure 4C and D), indicating that our nomogram was a more accurate method for OS prediction among ESCC patients after surgery.

The established nomogram was then verified in the 183 patients' validation cohort. In the validation cohort, the 3and 5-year survival rates predicted by the calibrated curve of the nomogram were consistent with real-world observations (Figure 5A and B). The AUC value for the nomogram was significantly higher than that for the TNM staging system (Figure 5C and D). Collectively, these results demonstrate that the nomogram was more accurate and effective for the prediction of survival among ESCC patients than existing methods.

\section{Discussion}

Currently, prognostic prediction and postoperative treatment plan establishment among ESCC patients are based primarily on the TNM staging system. However, due to tumor heterogeneity, differences in the prognosis of ESCC patients within the same TNM stage were observed. ${ }^{11}$ Some studies have confirmed that the nomogram model based on TNM stage can predict the prognosis of ESCC patients more accurately than TNM stage alone. ${ }^{7,12,13}$ Therefore, autologous prognostic indicators were combined with the TNM staging system to improve prognostic prediction accuracy among ESCC patients. In recent years, accumulating 
Table 2 Univariate and Multivariate Cox Regression Analyses for Overall Survival in Patients with ESCC in Original Queue

\begin{tabular}{|c|c|c|c|c|}
\hline \multirow[t]{2}{*}{ Variables } & \multicolumn{2}{|c|}{ Univariate Analysis } & \multicolumn{2}{|c|}{ Multivariate Analysis } \\
\hline & HR (95\% Cl) & $P$ value & HR (95\% Cl) & $P$ value \\
\hline Sex: Male vs Female & $0.83(0.62-1.10)$ & 0.194 & & \\
\hline Age: $>60$ vs $\leq 60$ & $1.12(1.00-1.27)$ & 0.050 & & \\
\hline Tumor location & & 0.989 & & \\
\hline Middle vs Upper & $1.00(0.62-1.63)$ & 0.994 & & \\
\hline Lower vs Upper & $0.98(0.59-1.63)$ & 0.945 & & \\
\hline Grade & & $<0.00 I^{*}$ & & $0.002 *$ \\
\hline Moderately vs Well & $3.64(1.48-8.92)$ & $0.005^{*}$ & $1.79(0.70-4.58)$ & \\
\hline Poorly vs Well & $5.85(2.40-14.27)$ & $<0.00 I^{*}$ & $2.63(1.04-6.72)$ & \\
\hline TNM stage (AJCC, 8th) & & $<0.00 I^{*}$ & & $<0.001 *$ \\
\hline II vs I & $2.86(1.80-4.54)$ & $<0.00 I^{*}$ & $2.36(1.45-3.84)$ & $0.00 I^{*}$ \\
\hline III vs I & $5.82(3.65-9.28)$ & $<0.00 I^{*}$ & $4.68(2.87-7.62)$ & $<0.001 *$ \\
\hline PLR: $>132$ vs $\leq 132$ & $1.55(1.22-1.97)$ & $<0.00 I^{*}$ & $1.44(1.11-1.86)$ & $0.006 *$ \\
\hline NLR: $>1.36$ vs $\leq 1.36$ & $1.39(1.06-1.81)$ & $0.017^{*}$ & I.II (0.84-I.46) & 0.474 \\
\hline MLR: $>0.27$ vs $\leq 0.27$ & $1.39(1.09-1.78)$ & $0.009 *$ & $1.24(0.95-1.63)$ & 0.108 \\
\hline AGR: $>1.75$ vs $\leq 1.75$ & $0.54(0.42-0.70)$ & $<0.00 I^{*}$ & $0.54(0.42-0.7 \mathrm{I})$ & $<0.001 *$ \\
\hline APS & & $<0.00 I^{*}$ & & $<0.001 *$ \\
\hline I vs 0 & $2.12(1.44-3.12)$ & $<0.00 I^{*}$ & $2.11(1.43-3.11)$ & $<0.001 *$ \\
\hline 2 vs 0 & $3.17(2.12-4.72)$ & $<0.00 I^{*}$ & $3.34(2.24-4.98)$ & $<0.001 *$ \\
\hline
\end{tabular}

Note: *Represents a statistically difference.

Abbreviations: ESCC, esophageal squamous cell carcinoma; TNM, tumor, node, metastasis; AJCC, American Joint Committee on Cancer; PLR, platelet-to-lymphocyte ratio; NLR, neutrophil-to-lymphocyte ratio; MLR, monocyte-to-lymphocyte ratio; AGR, albumin-globulin ratio; APS, AGR-PLR score.

evidence has demonstrated the key roles played by the chronic inflammatory response in tumorigenesis, invasion and metastasis for various tumor types. The associated inflammatory and nutritional indicators in peripheral blood, such as PLR, NLR, MLR and AGR, have been closely associated with the prognosis of patients with various types of tumors. ${ }^{7-10}$ Due to the advantages of high repeatability, high accuracy and low cost associated with the assessment of inflammation indicators in peripheral blood, the contributions of inflammatory and nutrition-related indicators in peripheral blood on tumors prognosis and diagnosis have attracted increasing attention.

Plasma albumin and globulin are two important components of human plasma that have been demonstrated to play important roles during the anti-tumor response. ${ }^{14}$ Reductions in plasma albumin levels serve as an indicator of poor nutritional status and the occurrences of chronic inflammatory reactions. In a variety of malignant tumors, including lung, nasopharyngeal and breast cancers, low albumin levels have been demonstrated to serve as an independent prognostic factor in patients. ${ }^{15,16}$ Plasma globulin is synthesized by immune organs, and the levels of plasma globulin directly reflect the status of inflammatory and immune responses. ${ }^{17}$ In the inflammatory microenvironment, plasma globulin levels are promoted by various inflammatory factors. As reported by previous studies, plasma globulin levels are significantly correlated with poor prognosis among patients with malignant tumors. $^{18,19}$ Recently, AGR (calculated as the ratio between albumin and globulin levels in the serum) has been identified as an important indicator for tumors evaluation, including ESCC. ${ }^{9,10,20}$ As reported by Oki et al, the preoperative reduction of AGR levels was associated with poor prognosis, increased tumor infiltration depth, and positive lymph node metastasis in ESCC patients. ${ }^{9}$ Poor OS IN patients with ESCC was found to be associated with low AGR levels. ${ }^{10,20}$ Consistently, in our study, we identified AGR to be an independent prognostic indicator for ESCC patients. Patients with low AGR levels presented with a shorter life span after surgery. 
Table 3 Baseline Characteristics for ESCC Patients with APS in Original Queue

\begin{tabular}{|c|c|c|c|c|c|}
\hline Clinical Parameter & APS $=0(86)$ & APS $=I(222)$ & APS=2 (142) & $\chi^{2}$ & $\boldsymbol{P}$ \\
\hline Sex & & & & 3.32 & 0.190 \\
\hline Male & 69 & 161 & 113 & & \\
\hline Female & 17 & 61 & 29 & & \\
\hline Age & & & & 1.10 & 0.576 \\
\hline$\leq 60$ & 47 & 113 & 80 & & \\
\hline$>60$ & 39 & 109 & 62 & & \\
\hline Histological grade & & & & 1.95 & 0.745 \\
\hline Well differentiated & 7 & 12 & 7 & & \\
\hline Moderately differentiated & 40 & 94 & 64 & & \\
\hline Poorly or not differentiated & 39 & 116 & 71 & & \\
\hline Tumor location & & & & 1.26 & 0.868 \\
\hline Upper & 4 & 17 & 9 & & \\
\hline Middle & 54 & 133 & 83 & & \\
\hline Lower & 28 & 72 & 50 & & \\
\hline T stage & & & & 3.26 & 0.775 \\
\hline TI & 22 & 50 & 34 & & \\
\hline $\mathrm{T} 2$ & 27 & 54 & 39 & & \\
\hline T3 & 36 & 112 & 65 & & \\
\hline $\mathrm{T} 4$ & $\mathrm{I}$ & 6 & 4 & & \\
\hline $\mathrm{N}$ stage & & & & 7.62 & 0.267 \\
\hline No & 49 & 122 & 70 & & \\
\hline $\mathrm{NI}$ & 26 & 69 & 39 & & \\
\hline N2 & 10 & 24 & 28 & & \\
\hline N3 & 1 & 7 & 5 & & \\
\hline TNM stage (AJCC, 8th) & & & & 1.88 & 0.758 \\
\hline I & 15 & 39 & 24 & & \\
\hline II & 46 & 104 & 65 & & \\
\hline III & 25 & 79 & 53 & & \\
\hline PLR & & & & 231.99 & $<0.001 *$ \\
\hline PLR $\leq 132$ & 86 & 127 & 0 & & \\
\hline PLR $>132$ & 0 & 95 & 142 & & \\
\hline NLR & & & & 11.25 & $0.004 *$ \\
\hline$N L R \leq 1.36$ & 35 & 76 & 30 & & \\
\hline$N L R>1.36$ & 51 & 146 & 112 & & \\
\hline MLR & & & & 5.07 & 0.079 \\
\hline$M L R \leq 0.27$ & 59 & 161 & 87 & & \\
\hline$M L R>0.27$ & 27 & 61 & 55 & & \\
\hline AGR & & & & 223.96 & $<0.001 *$ \\
\hline$A G R \leq 1.75$ & 0 & 127 & 142 & & \\
\hline$A G R>1.75$ & 86 & 95 & 0 & & \\
\hline
\end{tabular}

Note: *Represents a statistically difference.

Abbreviations: ESCC, esophageal squamous cell carcinoma; TNM, tumor, node, metastasis; AJCC, American Joint Committee on Cancer; PLR, platelet-to-lymphocyte ratio; NLR, neutrophil-to-lymphocyte ratio; MLR, monocyte-to-lymphocyte ratio; AGR, albumin-globulin ratio; APS, AGR-PLR score. 

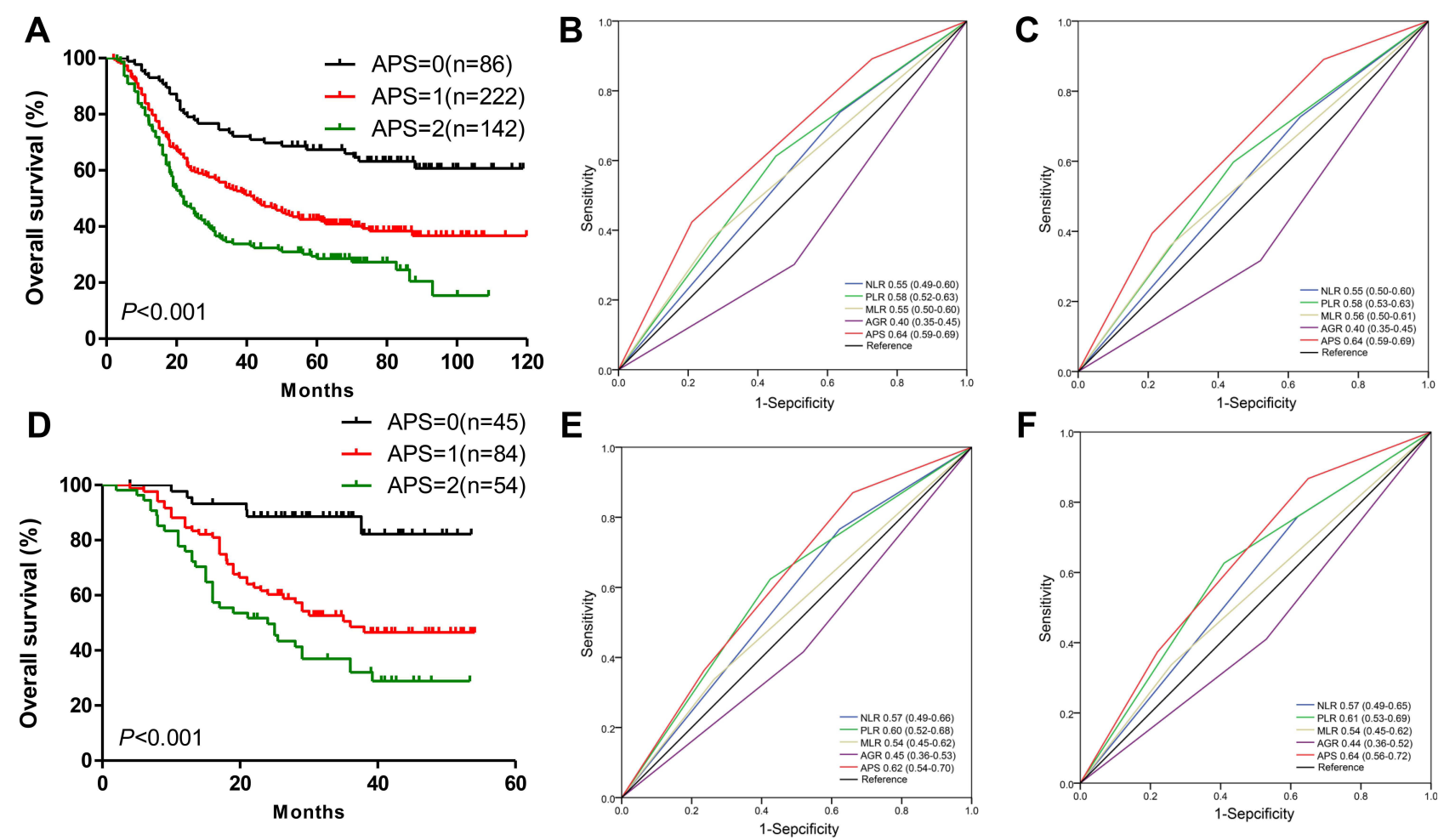

Figure 2 (A) The prognostic significance based on the APS among ESCC patients in the original cohort. (B and C) The predictive ability of the APS in ESCC was compared against the predictive abilities of PLR, NLR, MLR, and AGR by ROC curves in 3-years or 5-year in original cohort. (D) The prognostic significance is based on APS among ESCC patients in validation cohort. (E and F) The predictive ability of the APS in ESCC was compared against the predictive abilities of PLR, NLR, MLR, and AGR by ROC curves for 3-years or 5-year in validation cohort.

\section{Points}

$\begin{array}{lllllllllll}0 & 10 & 20 & 30 & 40 & 50 & 60 & 70 & 80 & 90 & 100\end{array}$

TNMstage

Grade

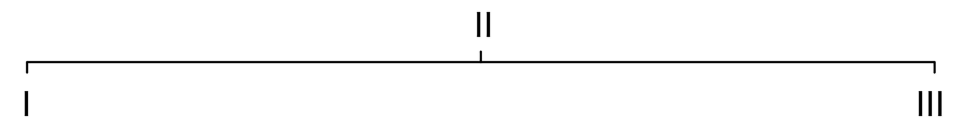

APS

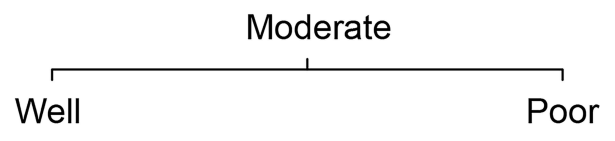

Total points

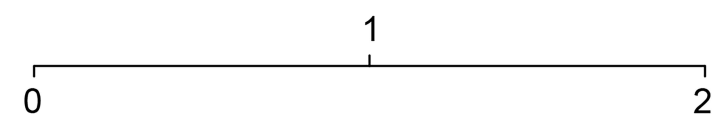

3-year survival

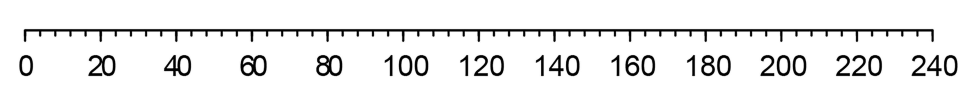

\section{5-year survival}
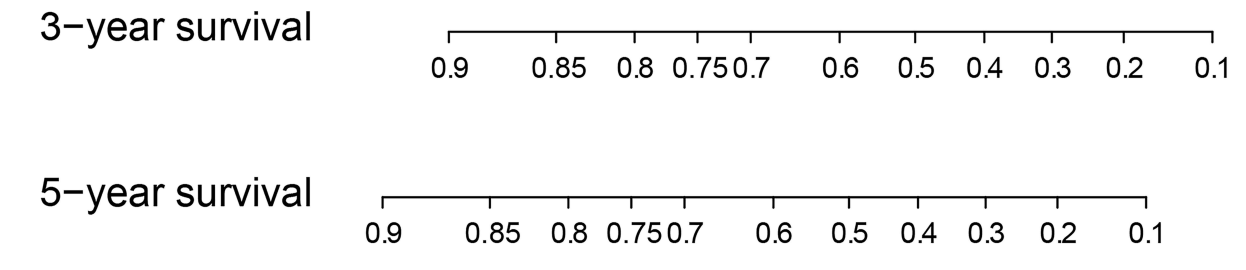

Figure 3 The nomogram integrating APS, TNM stage, and grade for the prediction of the 3- and 5-year survival rates of ESCC patients. 

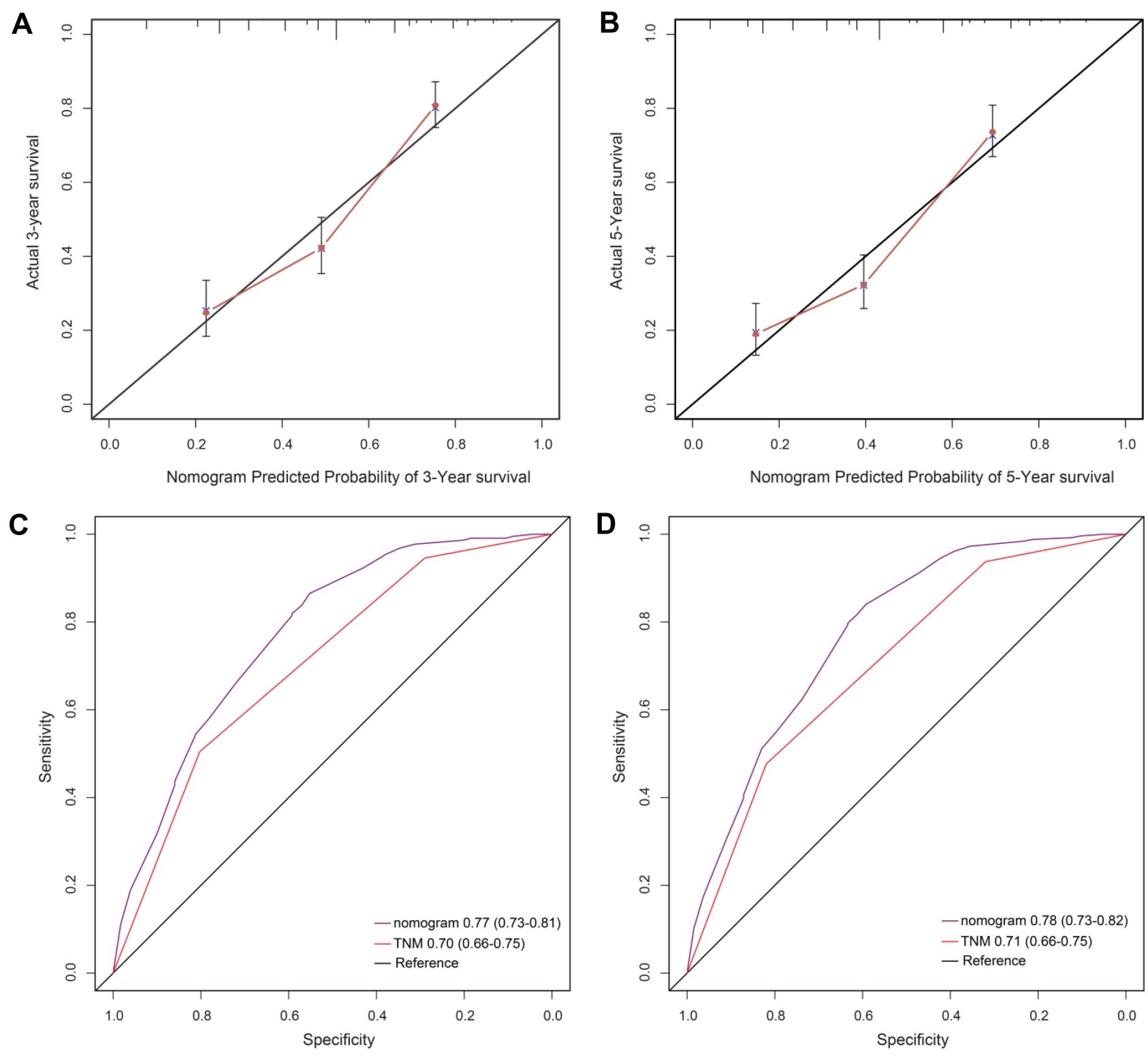

Figure 4 (A) The 3-year survival rate of ESCC patients predicted by the nomogram was highly consistent with the real-world, observed values in the original cohort. (B) The 5-year survival rate of ESCC patients predicted by the nomogram was highly consistent with the real-world, observed values in original cohort. (C) The ability of the nomogram to predict the 3-year survival rate of ESCC patients by ROC analysis, showing that the nomogram has a larger AUC than the TNM staging in original cohort. (D) The ability of the nomogram to predict the 5-year survival rate of ESCC patients by ROC analysis, showing that the nomogram has a larger AUC than TNM staging in original cohort.

Prior to this study, systemic inflammatory indicators including NLR, PLR, and MLR were reported to be key factors associated with ESCC prognosis. ${ }^{7,8}$ Xie et al found that poor survival prognosis among patients with Phase I and II ESCC was associated with high PLR. ${ }^{21}$ This association could potentially be explained by the promotion of tumor proliferation caused by the secretion of PDGF and TGF- $\beta$ from platelets. Rapid tumor proliferation, in turn, promotes compensatory hyperplasia among platelets. In addition, platelets and neutrophils also promote the adhesion and seeding of distant organs through the secretion of VEGF. ${ }^{22}$ In our study, we confirmed that both NLR and MLR were factors associated with ESCC survival, and PLR was identified as an independent prognostic factor for ESCC.

In this study, we established the APS system using PLR and AGR. The APS demonstrated better prognostic accuracy and effectiveness in ESCC patients than either PLR or AGR alone. In addition, a new nomogram for prognostic prediction was established according to tumor grade, APS and TNM stage. Compared with the traditional 8th version of the TNM staging system, this nomogram demonstrated improved 

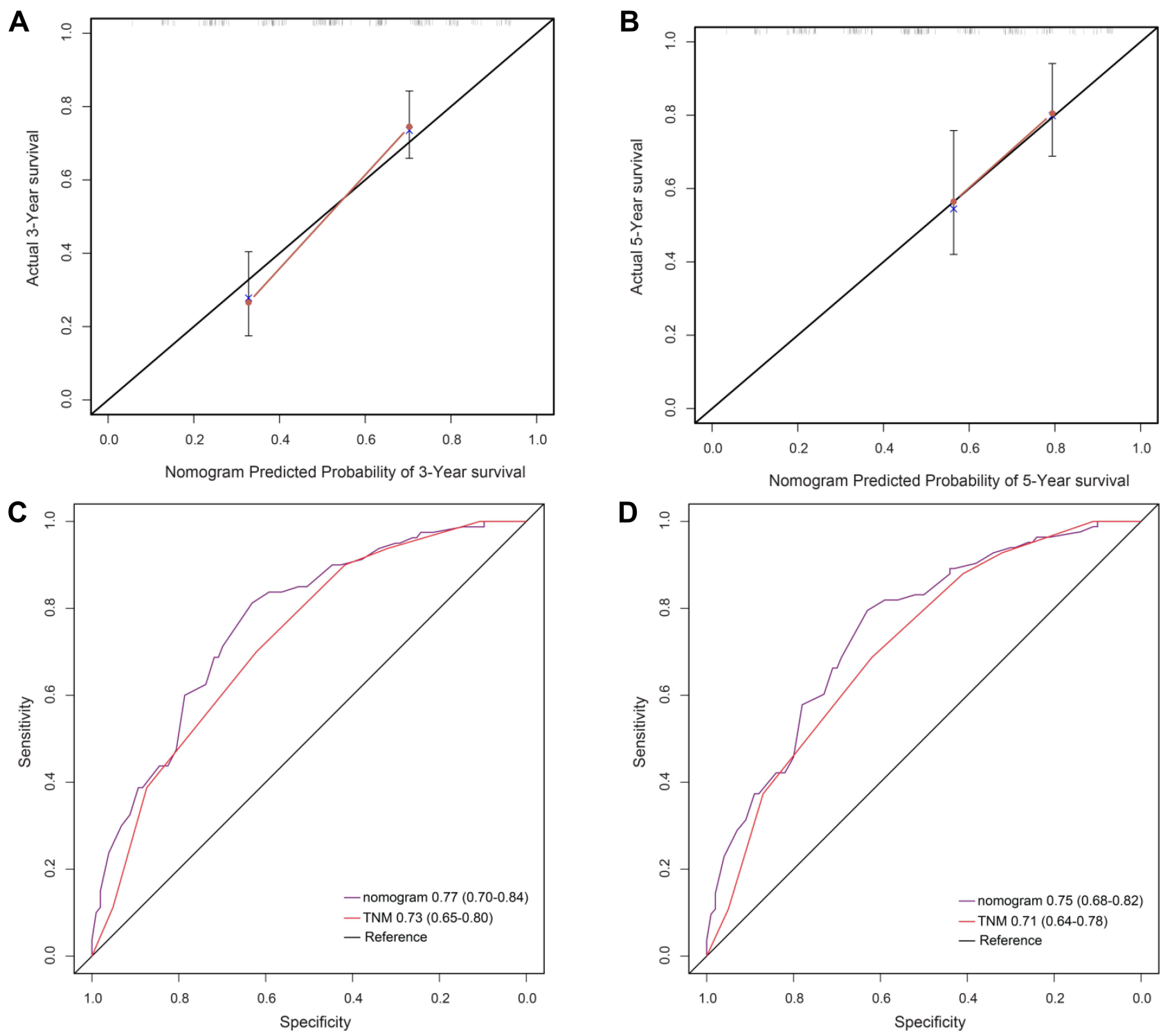

Figure 5 (A) The 3-year survival rate of ESCC patients predicted by the nomogram is highly consistent with the real-world, observed values in the validation cohort. (B) The 5-year survival rate of ESCC patients predicted by the nomogram is highly consistent with the real-world, observed values in the validation cohort. (C) The ability of the nomogram to predict the 3-year survival rate of ESCC patients by ROC analysis, showing that the nomogram has a larger AUC than the TNM staging in validation cohort. (D) The ability of the nomogram to predict the 5-year survival rate of ESCC patients by ROC analysis, showing that the nomogram has a larger AUC than the TNM staging in validation cohort.

sensitivity and specificity for the prediction of the 3- and 5-year survival. This result was subsequently confirmed using a separate validation cohort. As demonstrated by the calibrated nomogram in both the testing and validation cohorts, the predicted survival rate was highly consistent with the real-world observations. Therefore, we believed that APS and the APS-based prognostic nomogram represent promising effective and accurate indicators for survival prediction among ESCC patients.

In our results, we demonstrated the practical values of APS for prognostic prediction in ESCC patients. However, this result was limited for the following reasons. First, the data used to establish the nomogram were obtained from a single medical institution. Second, as is typical of retrospective studies, the potentials of selection bias, detection bias, and analysis bias are inevitable. Third, the cut-off values used in this study were not further validated. Fourth, the current standard treatment for operable $\mathrm{N}+$ or T3-4 locally advanced ESCC patients is neoadjuvant chemoradiotherapy plus surgery. However, our study includes a part of patients who need neoadjuvant treatment have undergone surgery alone. Our results maybe only suitable 
for operable esophageal cancer patients who have not undergone neoadjuvant chemoradiation. Therefore, further validation of the prognostic significance of APS in largescale prospective studies and across more pathological types of EC patients remains necessary.

\section{Conclusion}

APS is a novel independent prognostic indicator among patients after the radical resection of ESCC and serves as a potential biomarker for the monitoring of the therapeutic response. The prognostic nomogram that was established integrating APS was able to objectively and reliably predicted survival among ESCC patients after radical resection, with better performance than the traditional TNM staging system. Therefore, this nomogram could facilitate the distinction among different ESCC patients according to predicted survival outcome and allow for the early establishment of precise and timely individualized treatment strategies.

\section{Code Availability}

The authors declare that all data and R scripts supporting the findings of this study are available from the corresponding author upon reasonable request.

\section{Data Sharing Statement}

The data used to support the findings of this study are available from the corresponding author upon request.

\section{Ethics Statement}

The study protocol was performed in accordance with the guidelines outlined in the Declaration of Helsinki. The Ethics Committee of the First Affiliated Hospital of Soochow University approved the study, and all participants signed informed consent statements.

\section{Funding}

There is no funding to report.

\section{Disclosure}

The authors declare that they have no competing interests.

\section{References}

1. Bray F, Ferlay J, Soerjomataram I, Siegel RL, Torre LA, Jemal A. Global cancer statistics 2018: GLOBOCAN estimates of incidence and mortality worldwide for 36 cancers in 185 countries. CA Cancer J Clin. 2018;68(6):394-424. doi:10.3322/caac.21492

2. Huang FL, Yu SJ. Esophageal cancer: risk factors, genetic association, and treatment. Asian J Surg. 2018;41(3):210-215. doi:10.1016/j. asjsur.2016.10.005
3. Lin Y, Totsuka Y, He Y, et al. Epidemiology of esophageal cancer in Japan and China. J Epidemiol. 2013;23(4):233-242. doi:10.2188/jea. JE20120162

4. Yang H, Liu H, Chen $\mathrm{Y}$, et al. Neoadjuvant chemoradiotherapy followed by surgery versus surgery alone for locally advanced squamous cell carcinoma of the esophagus (NEOCRTEC5010): a Phase III multicenter, randomized, open-label clinical trial. J Clin Oncol. 2018;36(27):2796-2803. doi:10.1200/JCO.2018.79.1483

5. Zhang H, Chen X, Wang S, Fan J, Lu L. Poorer prognosis associated with simultaneous lymphatic and vascular invasion in patients with squamous carcinoma of the thoracic oesophagus. Eur J Cardiothorac Surg. 2017;52(2):378-384. doi:10.1093/ejcts/ezx081

6. Grivennikov SI, Greten FR, Karin M. Immunity, inflammation, and cancer. Cell. 2010;140(6):883-899. doi:10.1016/j.cell.2010.01.025

7. Shao Y, Ning Z, Chen J, et al. Prognostic nomogram integrated systemic inflammation score for patients with esophageal squamous cell carcinoma undergoing radical esophagectomy. Sci Rep. 2015;5:18811. doi:10.1038/srep18811

8. Geng Y, Shao Y, Zhu D, et al. Systemic immune-inflammation index predicts prognosis of patients with esophageal squamous cell carcinoma: a propensity score-matched analysis. Sci Rep. 2016;6:39482. doi:10.1038/srep39482

9. Oki S, Toiyama Y, Okugawa Y, et al. Clinical burden of preoperative albumin-globulin ratio in esophageal cancer patients. Am J Surg. 2017;214(5):891-898. doi:10.1016/j.amjsurg.2017.04.007

10. Zhang F, Sun P, Wang ZQ, et al. Low preoperative albumin-globulin score predicts favorable survival in esophageal squamous cell carcinoma. Oncotarget. 2016;7(21):30550-30560. doi:10.18632/oncotarget.8868

11. Burke HB. Outcome prediction and the future of the TNM staging system. J Natl Cancer Inst. 2004;96(19):1408-1409. doi:10.1093/ jnci/djh293

12. Guo X, Zhang H, Xu L, et al. Value of nomogram incorporated preoperative tumor volume and the number of postoperative pathologically lymph node metastasis regions on predicting the prognosis of thoracic esophageal squamous cell carcinoma. Cancer Manag Res. 2021;13:4619-4631. doi:10.2147/CMAR.S307764

13. Zheng Z, Lin D, Chen Q, et al. Prognostic value of combined detection of preoperative albumin-to-fibrinogen ratio and neutrophil-to-lymphocyte ratio in operable esophageal squamous cell carcinoma patients without neoadjuvant therapy. Cancer Manag Res. 2021;13:2359-2370. doi:10.2147/CMAR.S296266

14. Chen WZ, Yu ST, Xie R, Lv YX, Xu DB, Yu JC. Preoperative albumin/globulin ratio has predictive value for patients with laryngeal squamous cell carcinoma. Oncotarget. 2017;8(29):48240-48247. doi:10.18632/oncotarget. 18443

15. Gupta D, Lis CG. Pretreatment serum albumin as a predictor of cancer survival: a systematic review of the epidemiological literature. Nutr J. 2010;9:69. doi:10.1186/1475-2891-9-69

16. McMillan DC. Systemic inflammation, nutritional status and survival in patients with cancer. Curr Opin Clin Nutr Metab Care. 2009;12 (3):223-226. doi:10.1097/MCO.0b013e32832a7902

17. Ballow M. Mechanisms of action of intravenous immune serum globulin in autoimmune and inflammatory diseases. J Allergy Clin Immunol. 1997;100(2):151-157. doi:10.1016/S0091-6749(97)70217-3

18. Adly L, Hill D, Sherman ME, et al. Serum concentrations of estrogens, sex hormone-binding globulin, and androgens and risk of breast cancer in postmenopausal women. Int $J$ Cancer. 2006;119 (10):2402-2407. doi:10.1002/ijc.22203

19. Sawada N, Iwasaki M, Inoue M, et al. Plasma testosterone and sex hormone-binding globulin concentrations and the risk of prostate cancer among Japanese men: a nested case-control study. Cancer Sci. 2010;101(12):2652-2657. doi:10.1111/j.1349-7006.2010.01721.x

20. Li XH, Gu WS, Wang XP, et al. Low preoperative albumin-toglobulin ratio predict poor survival and negatively correlated with fibrinogen in resectable esophageal squamous cell carcinoma. J Cancer. 2017;8(10):1833-1842. doi:10.7150/jca.19062 
21. Xie X, Luo KJ, Hu Y, Wang JY, Chen J. Prognostic value of preoperative platelet-lymphocyte and neutrophil-lymphocyte ratio in patients undergoing surgery for esophageal squamous cell cancer. Dis Esophagus. 2016;29(1):79-85. doi:10.1111/dote.12296
22. Cools-Lartigue J, Spicer J, McDonald B, et al. Neutrophil extracellular traps sequester circulating tumor cells and promote metastasis. J Clin Invest. 2013;123(8):3446-3458. doi:10.1172/ JCI67484

\section{Publish your work in this journal}

Cancer Management and Research is an international, peer-reviewed open access journal focusing on cancer research and the optimal use of preventative and integrated treatment interventions to achieve improved outcomes, enhanced survival and quality of life for the cancer patient.
The manuscript management system is completely online and includes a very quick and fair peer-review system, which is all easy to use. Visit http://www.dovepress.com/testimonials.php to read real quotes from published authors. 